Publ. RIMS. Kyoto. Univ.

12 Suppl. (1977), 301-307.

\title{
Microlocal Calculus and Fourier Transforms
}

\author{
by \\ Masakazu Muro*
}

$\S 1$. In this note we shall explain the general scheme for calculation of the Fourier transforms of relatively invariant hyperfunctions on regular prehomogeneous vector spaces by Microlocal Calculus. The details of the theory will appear in [1], [3] and [6]. The principal purpose of this note is to state Theorem 6 .

A prehomogeneous vector space over the complex number field $\boldsymbol{C}$ is by definition a triple $(G, \rho, V)$ of an affine algebraic group $G$, a finite dimensional vector space $V$ and a linear representation $\rho$ of $G$ on $V$, all defined over $C$, such that there exists a point $x \in V$, (which is called a generic point of $V$ ), whose $G$-orbit $\rho(G) \cdot x$ is dense in $V$. A polynomial $f(x)$ on $V$ is called a relative invariant if $f(\rho(g) \cdot x)=\chi(g) \cdot f(x)$ for every $g \in G$, with $\chi$ denoting a rational character of $G$. It is a homogeneous polynomial and is uniquely determined by $\chi$ up to a constant factor. Further, $(G, \rho, V)$ is called regular if it possesses a relative invariant $f(x)$ whose logarithmic Hessian $\operatorname{det}\left(\partial^{2} \log f(x) / \partial x_{i} \partial x_{j}\right)$ does not vanish indentically. For example $(G, \rho, V)$ is known to be regular if $G$ and the isotropy group at a generic point of $V$ are both reductive. And we call it irreducible if $\rho$ is an irreducible representation. An irreducible regular prehomogeneous vector space has just one irreducible relative invariant up to a constant multiple.

Let $\mathfrak{g}$ be the Lie algebra of $G$, and $x \mid \rightarrow A \cdot x$ be the derived representation of $\rho$. Let $\mathcal{E}^{f}$ be the sheaf of microdifferential operators of finite order on the cotangent bundle $T^{*} V$. A good Lagrangian of $T^{*} V$ is by definition a Lagrangian submanifold which is a $G$-orbit of some $\left(x_{0}, y_{0}\right)$ $\in T^{*} V$, and contained in $W_{0}$, which is defined in [I] or [2]. Then we have the following theorem.

Received October 12, 1976.

* Department of Mathematics, Faculty of Science, Kyoto University, Kyoto 606. 
Theorem 1. Let $\mathcal{H}_{s}=\mathcal{E}^{\mathrm{s}} / \mathcal{g}[s]$ be the holonomic system of $f^{s}$, i.e., $g[s]=\left\{P\left(s, x, D_{x}\right) \in \mathcal{E}^{f}[s] ; P\left(s, x, D_{x}\right) f^{s}=0\right\} . \quad$ In a neighborhood of a generic point of a good Lagrangian, $g[s]$ is generated by $\{\langle A \cdot x$, $\left.D_{x}>-s \delta \chi(A) ; A \in \mathfrak{g}\right\}$. In the real locus, this holonomic system has a microfunction solution $s p\left(|f(x)|^{s}\right)$.

The principal symbol (which is defined later) on the conormal bundle of the origin is represented by the Fourier transeform of $|f(x)|^{s}$. So we have only to seek for the principal symbol on it. In the following sections we shall explain the calculation of the principal symbols on good Lagrangians and their relations. The details of the theory of prehomogeneous vector spaces can be found in [1], [4], [5] and [7].

$\S 2$. Let $(G, \rho, V)$ be an $n$-dimensional irreducible regular prehomgeneous vector space whose relative invariant is $f(x)$ with a character $\chi$, and degree of $f(x)$ is $r$. Then we can define another regular prehomogeneous vector space $\left(G, \rho^{*}, V^{*}\right)$, where $V^{*}$ is the dual space of $V$ and $\rho^{*}$ is the contragredient representation. We know that it has also only one relative invariant $f^{*}(y)$ with character $\chi^{-1}$. Let $\left(G_{\boldsymbol{R}}^{+}, \rho, V_{\boldsymbol{R}}\right)$ and $\left(G_{\boldsymbol{R}}^{+}, \rho^{*}, V_{\boldsymbol{R}}^{*}\right)$ be real forms of $(G, \rho, V)$ and $\left(G, \rho^{*}, V^{*}\right)$, respective1y. We put $\bigsqcup_{i=1}^{l} V_{i}=V_{\boldsymbol{R}}-S$ and $\bigsqcup_{i=1}^{l} V_{i}^{*}=V_{\boldsymbol{R}}^{*}-S^{*}$ as decompositions into connected components, where $S=\{x \in V ; f(x)=0\}$ and $S^{*}=\left\{y \in V^{*}\right.$; $\left.f^{*}(y)=0\right\}$.

\section{Definition 2.}

$$
\begin{gathered}
|f|_{i}^{s}(x)=\left\{\begin{array}{cc}
|f(x)|^{s} & x \in V_{i} \\
0 & x \notin V_{i} .
\end{array}\right. \\
\left|f^{*}\right|_{i}^{s}(y)=\left\{\begin{array}{cc}
\left|f^{*}(y)\right|^{s} & y \in V_{i}^{*} \\
0 & y \notin V_{i}^{*} .
\end{array}\right.
\end{gathered}
$$

Then each $|f|_{i}^{s}(x)$ is a hyperfunction on $V_{\boldsymbol{R}}$, more precisely, a microfunction on $\sqrt{-1} T^{*} V_{\boldsymbol{R}}$, and this microfunction satisfies the holonomic system $\mathscr{M}_{s}$ for generic $s \in C$. 
Let $\Lambda$ be an irreducible component of $\widetilde{S S}\left(\mathcal{M}_{s}\right)$, and $\Lambda^{\boldsymbol{R}}$ be its real locus on $\sqrt{-1} T^{*} V_{\boldsymbol{R}}$, which is a real Lagrangian submanifold in $\sqrt{-1} T^{*} V^{\boldsymbol{R}}$. Assume that the symbol ideal $J$ of $g[s]$ is a reduced ideal in a neighborhood of a generic point $p$ of $\Lambda$. Then the dimension of microfunction solution space is one. We say that this Lagrangian submanifold is simple and that $\mathscr{M}_{s}$ is simple characteristic along $\Lambda$. Let $\Lambda_{1}, \Lambda_{2}$ be two simple Lagrangian submanifolds of $\mathscr{M}_{s}$. Suppose that in a neighborhood of $p \in \Lambda_{1}^{\boldsymbol{R}} \cap \Lambda_{2}^{\boldsymbol{R}}, \mathscr{M}_{s}$ is isomorphic microlocally to

$$
\mathscr{M}_{\lambda} ;\left(x_{1} \mathrm{D}_{x_{1}}-\lambda\right) u=0, \quad x_{2} u=0, \cdots, x_{n} u=0
$$

where $\lambda+1=\operatorname{ord}_{\Lambda_{1}} f^{s}-\operatorname{ord}_{\Lambda_{2}} f^{s}+\frac{1}{2}$, and $\Lambda_{1}=\left\{y_{1}=x_{2}=\cdots=x_{n}=0\right\}, \Lambda_{2}=\left\{x_{1}\right.$ $\left.=x_{2}=\cdots=x_{n}=0\right\}$, by a suitable real quantized contact transformation. Then, in a neighborhood of $p, \Lambda_{1}^{\boldsymbol{R}}=\Lambda_{1}{ }^{+} \cup^{-} \Lambda_{1}{ }^{-}$and $\Lambda_{2}^{\boldsymbol{R}}=\Lambda_{2}{ }^{+}\left[\Lambda_{2}{ }^{-}\right.$. On each connected component, there is only one dimensional solution space. Moreover these solutions have relations one another. We shall explain this more precisely in the next section. We can calculate the Fourier transform of $f^{s}(x)$ by using such relations. For this purpose, we have to introduce real principal symbols, which is the real analytic section of a line bundle on $\Lambda$.

§3. Real principal symbols of microfunction solutions of holonomic systems was introduced by Prof. M. Kashiwara. The real principal symbol of a microfunction $\mathrm{P}\left(x^{\prime \prime}, \mathrm{D}_{x^{\prime}}\right) \delta\left(x_{1}\right) \delta\left(x_{2}\right) \cdots \delta\left(x_{k}\right)$ is defined to be

$$
\sigma(P)\left(x^{\prime \prime}, \xi^{\prime}\right) \sqrt{\frac{\left|d \xi_{1} \wedge d \xi_{2} \wedge \cdots \wedge d \xi_{k} \wedge d x_{k+1} \wedge \cdots \wedge d x_{n}\right|}{\left|d x_{1} \wedge d x_{2} \cdots \wedge d x_{n}\right|}}
$$

when we use the coordinate $\sqrt{-1} T^{*} V_{\boldsymbol{R}}=\left(x_{1}, x_{2}, \cdots, x_{n} ; \sqrt{-1} \xi_{1}, \sqrt{-1} \xi_{2}\right.$, $\left.\cdots, \sqrt{-1} \xi_{n}\right)$ such that $\Lambda=\left\{x^{\prime}=\xi^{\prime \prime}=0\right\}$, where $x^{\prime}=\left(x_{1}, x_{2}, \cdots, x_{k}\right), x^{\prime \prime}$ $=\left(x_{k+1}, \cdots, x_{n}\right), \xi^{\prime}=\left(\xi_{1}, \cdots, \xi_{k}\right)$ and $\xi^{\prime \prime}=\left(\xi_{k+1}, \cdots, \xi_{n}\right)$. Here $\mathrm{P}\left(x^{\prime \prime}, D_{x^{\prime}}\right)$ is a microdifferential operator of fractional order. It is an analytic section of $\left|\Omega_{A}\right|^{1 / 2} \otimes\left|\Omega_{V}\right|^{-1 / 2}$ where $\left|\Omega_{V}\right|$ and $\left|\Omega_{A}\right|$ are sheaves of real analytic volume elements on $V$ and $\Lambda$, respectively. Moreover, it is independent of the choice of coordinate systems. When $\varphi(y)$ is a homogeneous hyperfunction, the principal symbol of $\psi(x)=\int \varphi(y) \exp \sqrt{-1}\langle y, x\rangle d y$ at the conormal bundle of the origin can be easily calculated. 
Theorem 3. Suppose that $\varphi(y)$ is a homogeneous hyperfunction of homogeneous degree $\nu$ on $\boldsymbol{R}^{n}$ which satisfies the following conditions

I) $\varphi(y)$ is real analytic in an open cone $\Gamma \subset \boldsymbol{R}^{n}$.

II) $\operatorname{Supp}(\varphi(y)) \subset \bar{\Gamma}$

Then we have the following principal symbol on the conormal bundle of the origin.

$$
\sigma(\psi(x))=\left(\frac{1}{2 \pi}\right)^{n / 2} \varphi(y) \sqrt{\frac{|d y|}{|d x|}}
$$

Therefore, if we know that the Fourier transform of a homogeneous hyperfunction $\varphi(x)$ is also a homogeneous hyperfunction, then we see that to calculate the Fourier transform of $\varphi(x)$ is nothing but to seek for the principal symbol of $\varphi(x)$ at the conormal bundle of the origin if $\breve{S S}(\varphi(x))$ contains it.

§4. Let $\Lambda$ be an irreducible component of $\check{S S}\left(\mathscr{M}_{s}\right)$, and $\bigsqcup_{i} \Lambda_{i}$ be a connected component decomposition of real locus of $\Lambda$. Then we can define a basis of real principal symbol on each $\Lambda_{i}$ as follows.

$$
\sigma_{\Lambda_{1}}\left(u_{s}\right)=\left|f_{\Lambda}\right| s \sqrt{\left|\omega_{A}\right| /|d x|}
$$

Here we set

$$
f_{\Lambda}=f \circ \pi /\left.s^{m_{\Lambda}}\right|_{\Lambda} . \quad \omega_{\Lambda}=\frac{\pi_{*}(d x) \bigwedge d s}{s^{l_{\Lambda}}}|d s|_{\Lambda} .
$$

$\pi$ is a projection map

$$
W=\left\{\left(x, s . \operatorname{grad}_{x} \log f(x)\right) \in T^{*} V ; x \in V-S\right\} \rightarrow V,
$$

and $s=\langle x, y\rangle / r$, ord $f^{s}=-m_{A} s-l_{A}$.

Then the real principal symbols of $|f|^{s}(x)$ on each $\Lambda_{i}$ can be expressed as follows.

$$
\sigma_{\Lambda_{i}}\left(f^{s}\right)=c_{\Lambda_{i}}(s)\left|f_{\Lambda}\right|^{s} \sqrt{\left|\omega_{\Lambda}\right| /|d x|}
$$

Definition 4. Let $\Lambda^{1}$ and $\Lambda^{2}$ be two irreducible components of $\check{S} S\left(\mathcal{M}_{s}\right)$, and $\bigsqcup_{i} \Lambda_{i}{ }^{1}$ and $\bigsqcup_{j} \Lambda_{j}{ }^{2}$ be connected component decompositions 
of them. We call $\left(c_{\Lambda_{i^{1}}}(s) / c_{\Lambda_{j^{2}}}(s)\right)$ the relation number between $\Lambda_{i}{ }^{1}$ and $\Lambda_{j}{ }^{2}$, and $c_{\Lambda_{i}}(s)$ are called the associated numbers.

Theorem 5. Let $\Lambda_{0}^{j}=V_{j} \times\{0\}$ and $\Lambda_{1}{ }^{i}=\{0\} \times V_{i}^{*}$. Let $A(s)$ be a matrix whose $\mathrm{i}-j$ entry is $c_{\Lambda_{1} i}(s) / c_{\Lambda_{0} j}(s)$. Then

$$
\left[\begin{array}{c}
|f|_{1}^{s}(x) \\
\vdots \\
|f|_{l}^{s}(x)
\end{array}\right]=(2 \pi)^{-n / 2}\left|c_{0}\right|^{s}\left|c_{1}\right|^{1 / 2} A(s) \int\left[\begin{array}{c}
\left|f^{*}\right|_{1}{ }^{-s-n / r}(y) \\
\vdots \\
\left|f^{*}\right|_{l^{-s-n / r}}(y)
\end{array}\right] \exp \sqrt{-1}\langle x, y\rangle d y
$$

where

$$
\begin{aligned}
& c_{0}=f^{*}(y) \cdot f\left(\operatorname{grad}_{y} \log f^{*}(y)\right) \\
& c_{1}=f^{*}(y)^{2 n / r} \operatorname{Hess}_{y} \log f^{*}(y) .
\end{aligned}
$$

This theorem can be easily proved by the general theory of prehomogeneous vector space. The problem is how to obtain $A(s)$.

$\S 5$. Let $\left(G^{\prime}, \rho^{\prime}, V^{\prime}\right)$ be another $n^{\prime}$-dimensional irreducible regular prehomogeneous vector space whose relative invariant is $f^{\prime}\left(x^{\prime}\right)$ with a rational character $\chi^{\prime}$. Let $\left(G^{\prime}, \rho^{* \prime}, V^{* \prime}\right)$ be the regular prehomogeneous vector space by the contragredient representation and $f^{* \prime}\left(y^{\prime}\right)$ be a relative invariant with a character $\chi^{\prime-1}$. Moreover, we assume the following conditions hold.

1) Let $\mathcal{M}_{s^{\prime}}^{\prime}=\mathcal{E}^{f}{ }_{v^{\prime}} f^{\prime s^{\prime}}\left(x^{\prime}\right)$ and in a neighborhood of $\left(x_{0}, y_{0}\right) \in T^{*} V$, $\mathcal{M}_{s}$ is isomorphic to $\mathcal{M}_{s^{\prime}}^{\prime} \hat{\otimes} \delta\left(x^{\prime \prime}\right)$

2) Let $\bigsqcup_{i=1}^{k} V_{i}^{\prime}=V_{\mathbb{R}}^{\prime}-S^{\prime}$ and $\bigsqcup_{j=1}^{k} V_{j}^{*}=V_{\boldsymbol{R}}^{* \prime}-S^{* \prime}$ be connected component decompositions where $S^{\prime}=\left\{x^{\prime} \in V^{\prime} ; f^{\prime}\left(x^{\prime}\right)=0\right\}$ and $S^{* \prime}=\left\{y \in V^{* \prime}\right.$; $\left.f^{* \prime}\left(y^{\prime}\right)=0\right\}$. We set

$$
\begin{aligned}
& \left|f^{\prime}\right|_{i}^{s}\left(x^{\prime}\right)=\left\{\begin{array}{ccc}
\left|f^{\prime}\left(x^{\prime}\right)\right|^{s} & \text { if } & x^{\prime} \in V_{i}^{\prime} \\
0 & \text { if } & x^{\prime} \notin V_{i}^{\prime}
\end{array}\right. \\
& \left|f^{* \prime}\right|{ }_{j}^{s}\left(y^{\prime}\right)=\left\{\begin{array}{ccc}
\left|f^{* \prime}\left(x^{\prime}\right)\right|^{s} & \text { if } & y^{\prime} \in V_{j}^{* \prime} \\
0 & \text { if } & y^{\prime} \notin V_{j}^{* \prime}
\end{array}\right.
\end{aligned}
$$

Then we have

$$
\left[\begin{array}{c}
\left|f^{\prime}\right|_{1}^{s}(x) \\
\vdots \\
\left|f^{\prime}\right|_{k}^{s}(x)
\end{array}\right]=(2 \pi)^{-n^{\prime} / 2}\left|c_{0}\right|_{\mid}^{s} \mid c_{1}^{\prime} i^{1 / 2 t} B\left(s^{\prime}\right) \int\left[\begin{array}{c}
\left|f^{* \prime}\right|_{1}^{-s-n / r}\left(y^{\prime}\right) \\
\vdots \\
\left|f^{* \prime}\right|_{k}{ }^{-s-n / r}\left(y^{\prime}\right)
\end{array}\right] \exp \sqrt{-1}\left\langle x^{\prime}, y^{\prime}\right\rangle d y
$$


where

$$
\begin{aligned}
& B\left(s^{\prime}\right) \text { an } k \times k \text { matrix } \\
& c_{0}^{\prime}=f^{* \prime}\left(y^{\prime}\right) f^{\prime}\left(\operatorname{grad}_{y^{\prime}} \log f^{* \prime}\left(y^{\prime}\right)\right) \\
& c_{1}^{\prime}=f^{* \prime}\left(y^{\prime}\right)^{2 n^{\prime} / r^{\prime}} \text { Hess }_{y^{\prime}} \log f^{* \prime}\left(y^{\prime}\right)
\end{aligned}
$$

3) Let $\Lambda=T_{G \cdot x_{0}}^{*} V$ and $\Lambda^{\prime}=T_{G \cdot y_{0}}^{*} V^{*}$ be two irreducible components of $\widetilde{S S}\left(\mathcal{M}_{s}\right)$. Let $\bigsqcup_{i=1}^{k} \Lambda_{i}^{\prime}=\Lambda_{\boldsymbol{R}}^{\prime}-\Sigma$ and $\bigsqcup_{j=1}^{k} \Lambda_{j}=\Lambda_{R}-\Sigma^{\prime}$ be connected component decompositions in a neighborhood of $\left(x_{0}, y_{0}\right)$ such that by a suitable real quantized contact transformation $\Phi$

$$
\begin{aligned}
& \Phi\left(\left(x_{0}, y_{0}\right)\right)=\left(0 ; d x_{n}\right) \\
& \Phi\left(\Lambda_{i}^{\prime}\right)=\mathrm{V}_{i}{ }^{\prime} \times \sqrt{-1}\left\{\left(y^{\prime \prime}\right)\right\} \\
& \Phi\left(\Lambda_{j}\right)=V_{j}{ }^{*} \times \sqrt{-1}\left\{\left(y^{\prime}\right)\right\}
\end{aligned}
$$

Here, $\Sigma$ and $\Sigma^{\prime}$ are the sets of non generic points of $\Lambda_{\boldsymbol{R}}$ and $\Lambda_{\boldsymbol{R}}^{\prime}$, respectively, and we set

$$
\begin{array}{ll}
x^{\prime}=\left(x_{1}, \cdots, x_{n^{\prime}}\right) & y^{\prime}=\left(y_{1}, \cdots, y_{n^{\prime}}\right) \\
x^{\prime \prime}=\left(x_{n^{\prime}+1}, \cdots, x_{n}\right) & y^{\prime \prime}=\left(y_{n^{\prime}+1}, \cdots, y_{n}\right) .
\end{array}
$$

Under these conditions, we have the following theorem.

Theorem 6. The matrix of relation numbers between $\Lambda^{\prime}$ and $\Lambda$ is

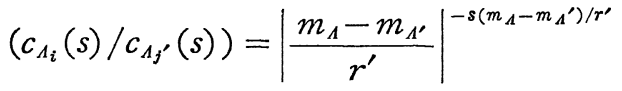

$$
\begin{aligned}
& \times B(s) \times\left[\begin{array}{c}
\exp (\pi / 4) \sqrt{-1}\left(\tau\left(\Lambda_{1}^{\prime}\right)-\tau\left(\Lambda \cap \Lambda^{\prime}\right)\right) \\
\dot{\theta} \cdot \operatorname{lin}(\pi / 4) \sqrt{-1}\left(\tau\left(\Lambda_{k}{ }^{\prime}\right)-\tau\left(\Lambda \cap \Lambda^{\prime}\right)\right)
\end{array}\right]
\end{aligned}
$$

where;

$$
\begin{array}{ll}
\text { i) } \quad \operatorname{ord}_{\Lambda^{\prime}} f^{s}=-s m_{\Lambda^{\prime}}-l_{\Lambda^{\prime}} \\
& \operatorname{ord}_{\Lambda} f^{s}=-s m_{\Lambda}-l_{\Lambda} \\
& s^{\prime}=-s\left(m_{\Lambda^{\prime}}-m_{\Lambda}\right)-\left(l_{\Lambda^{\prime}}-l_{\Lambda}\right)-n^{\prime} / r^{\prime} \\
\text { ii) } \quad \tau\left(\Lambda_{i}^{\prime}\right)=\operatorname{sgn}_{A \in \mathbf{g}}\left\langle A \cdot x_{i},-{ }^{t} A \cdot y_{i}\right\rangle \\
& \tau\left(\Lambda \cap \Lambda^{\prime}\right)=\underset{A \in \mathfrak{g}}{\operatorname{sgn}}\left\langle A \cdot x_{0},-{ }^{t} A \cdot y_{0}\right\rangle
\end{array}
$$


$\left(x_{i}, \sqrt{-1} y_{i}\right)$ is a generic point of $\Lambda_{i}{ }^{\prime}$.

Especially when $f^{\prime}\left(x^{\prime}\right)=x_{1}$, we have the following formula.

\section{Corollary 7.}

$$
\begin{aligned}
& \left(c_{\Lambda_{i}}(s) / c_{\Lambda_{j^{\prime}}}(s)\right)=\left|m_{\Lambda}-m_{A^{\prime}}\right|^{-s\left(m_{\Lambda}-m_{A^{\prime}}\right)-\left(l_{\left.\Lambda^{-}-l_{A^{\prime}}\right)}\right.} \\
& \quad \times \frac{\Gamma(\lambda+1)}{\sqrt{2 \pi}}\left[\begin{array}{l}
\exp (-\pi \sqrt{-1}(\lambda+1) / 2), \exp (\pi \sqrt{-1}(\lambda+1)) \\
\exp (\pi \sqrt{-1}(\lambda+1) / 2), \exp (-\pi \sqrt{-1}(\lambda+1))
\end{array}\right] \\
& \quad \times\left[\begin{array}{r}
\exp \left(\pi \sqrt{-1}\left(\tau\left(\Lambda_{1}^{\prime}\right)-\tau\left(\Lambda \cap \Lambda^{\prime}\right)\right) / 4\right), \\
, \exp \left(\pi \sqrt{-1}\left(\tau\left(\Lambda_{2}^{\prime}\right)-\tau\left(\Lambda \cap \Lambda^{\prime}\right)\right) / 4\right)
\end{array}\right],
\end{aligned}
$$

where $\lambda=-s\left(m_{\Lambda^{\prime}}-m_{\Lambda}\right)-\left(l_{\Lambda^{\prime}}-l_{\Lambda}\right)+1 / 2$.

By using this theorem, we can calculate $A(s)$. In this way, we have calculated many examples of Fourier transforms, and they can be found in [1], [3], and [6]. And we can find more "generalized" formula of this theorem in [1].

\section{References}

[1] Kashiwara, M., Kimura, T. and Muro, M., Microlocal calculus of simple holonomic systems and its applications, (to appear).

[2] Kashiwara, M. and Miwa, T., Microlocal calculus and Fourier transformation of relative invariants of prehomogeneous vector space, Surikaiseki kenkyujo kokyuroku. 238 (1975), 60-147, (in Japanese).

[3] Muro, M., in preparation.

[4] Sato, M. and Shintani, T., On zeta functions associated with prehomogeneous vector spaces, Ann. of Math., 100 (1974), 131-170.

[5] Sato, M. and Kimura, T., A classification of irreducible prehomogeneous vector spaces and their relative invariants. (to appear).

[6] Suzuki, T., in preparation.

[7] Sato, M. and Shintani, T., Theory of prehomogeous vector spaces, Sugaku no ayumi, (1970), (in Japanese). 
